\title{
PREVALÊNCIA DA DOENÇA RENAL CRÔNICA NOS ESTÁGIOS 3, 4 E 5 EM ADULTOS
}

Rita Maria Rodrigues Bastos*1, Marcus Gomes Bastos², Luís Cláudio Ribeiro², Ricardo Villela Bastos ${ }^{3}$, Maria Teresa Bustamante Teixeira ${ }^{2}$

Trabalho realizado na Universidade Federal de Juiz de Fora, Juiz de Fora, MG

\author{
*Correspondência: \\ Rita Maria Rodrigues Bastos \\ Rua Vinte e Um de Abril, 74 \\ - Juiz de Fora - MG \\ CEP: $36020-090$ \\ ritamrb@terra.com.br
}

\begin{abstract}
RESUMO
INTRODução. Medidas que otimizem a detecção precoce da doença renal crônica (DRC) são fundamentais para o retardo na evolução da doença e diminuição do aporte de indivíduos às terapias renais de substituição.

OBjetivos. Estimar a prevalência da DRC em seus estágios 3, 4 e 5, utilizando registros laboratoriais de indivíduos submetidos a dosagem de creatinina sérica por causas diversas em laboratório da rede particular do municíio de Juiz de Fora, no período de 2004 e 2005.

Métodos. O estudo foi consubstanciado pelo cálculo estimado da filtração glomerular, utilizando a equação do estudo MDRD (Modification of diet in renal disease), e seguiu os critérios propostos pelo K/DOQI (Kidney Disease Outcomes Quality Initiative) para o diagnóstico e classificação da DRC.

Resultados. A prevalência encontrada foi de $9,6 \%$, sendo $12,2 \%$ no sexo feminino, 5,8\% no sexo masculino, $3,7 \%$ em indivíduos abaixo de 60 anos e 25,2\% acima de 60 anos.

Conclusão. Os resultados evidenciam a prevalência da DRC não apenas como um indicador epidemiológico, mas demonstram um aspecto operacional alternativo para otimizar a capacidade de detecção dos casos e permitem sugerir a inclusão do cálculo da filtração glomerular como um dado complementar aos resultados das dosagens de creatinina sérica fornecidos pelos laboratórios.
\end{abstract}

Unitermos: Doença renal crônica. Filtração glomerular. Prevalência. Diagnóstico precoce.

\section{INTRODUÇÃO}

As alterações no perfil de morbimortalidade da população mundial, ocorrido nas últimas décadas, evidenciaram um aumento das doenças crônico degenerativas e projetaram a doença renal crônica (DRC) no cenário mundial como um dos maiores desafios à saúde pública deste século, com todas as suas implicações econômicas e sociais. 0 crescimento da população idosa e da prevalência de obesidade levou a um aumento das doenças crônicas, com destaque para o diabetes mellitus e a hipertensão arterial, principais causas de falência renal em todo o mundo'. A vigilância é parte fundamental para conter o aumento da endemia, visto que a expressão clínica das doenças crônicas não transmissíveis, em geral faz-se após longo tempo de exposição aos fatores de risco e da convivência assintomática do indivíduo com a doença não diagnosticada2,3,4.

Desde 2002, a Organização Mundial de Saúde vem recomendando aos diversos países a implementação da vigilância para doenças crônicas não transmissíveis, com enfoque nos fatores de risco que predizem as mais comuns delas 3,5 . O reconhecimento da DRC nos estágios iniciais e o encaminhamento precoce ao nefrologista são fundamentais para o retardo na evolução da doença e para a diminuição do aporte de indivíduos às terapias renais de substituiçãó6.
Embora muitos trabalhos estejam sendo realizados no sentido de se identificar a DRC, os estudos de base populacional ainda são escassos e muitas das prevalências estimadas indiretamente ${ }^{7,8}$, procedentes de casuísticas compostas por familiares de doentes renais crônicos e indivíduos pertencentes aos tradicionais grupos de risco para as doenças cardiovasculares 9 ,10,11.

Há poucos estudos disponíveis na literatura sobre a prevalência da doença renal no Brasil. Em São Paulo, realizou-se um estudo da função renal em idosos, em que foram encontrados hematúria em 26\% dos casos e proteinúria em $5 \%{ }^{12}$. Em Ibura, bairro da cidade de Recife-PE, detectou-se prevalência de alterações urinárias em 36\% da população e a persistência das alterações foi detectada em 10,7\% dos $\operatorname{casos}^{13}$. A hipercreatinemia fundamentou dois estudos de base populacional sobre a prevalência da DRC no Brasil. Lessa et al $1^{5}$, em Salvador- BA, avaliaram indivíduos acima de 20 anos de idade e, com base na creatinina sérica $(s c r)>1,3 \mathrm{mg} / \mathrm{dl}$, encontraram uma prevalência de 3, I \% de disfunção renal na população global. Passos et al ${ }^{14}$, em Bambuí, Minas Gerais, também com base na SCR, citam prevalência inexpressiva da disfunção renal na população abaixo de 60 anos e de $5,29 \%$ e $8,19 \%$ para os idosos do sexo feminino e masculino, respectivamente. 
Os critérios utilizados para a identificação da DRC nos estudos de rastreamento em populações diferem tanto pela escolha da população a ser rastreada quanto pelos parâmetros utilizados para identificar a presença e persistência da doença renal. As diretrizes propostas pelo Kidney Disease Outcomes Quality Initiative (K/DOQI,2002) estabelecem que a permanência da alteração renal deva persistir por no mínimo três meses, para que o diagnóstico da DRC seja estabelecido. Devido a ainda recente recomendação destas diretrizes, poucos foram os estudos em que este critério foi adotado, a despeito de sua importância para se excluir uma possível queda transitória da função renal ${ }^{15}$.

Este trabalho teve como objetivo estimar a prevalência da doença renal crônica, nos estágios 3, 4 e 5 em segmento da população adulta, submetida a exames laboratoriais por causas diversas em laboratório da rede particular do município de Juiz de Fora, MG, no período de janeiro de 2004 a dezembro de 2005. Além disso, analisamos a prevalência da DRC distribuída por sexo, faixa etária, estágio e pelas alterações de creatinina.

\section{Métodos}

Este trabalho foi realizado por meio de uma análise dos registros de usuários de um laboratório da rede particular do município de Juiz de Fora, MG, no período de janeiro de 1995 a dezembro de 2005.

O laboratório pertence à rede particular do Município de Juiz de Fora e não é conveniado ao Sistema Único de Saúde. É certificado pela norma ISO 9000 (2000) e pelo PALC (Programa de Acreditação para Laboratórios Clínicos).

Foram disponibilizados, de indivíduos não hospitalizados, os seguintes dados referentes ao período de janeiro de 1995 a dezembro de 2005: Ordem de Serviço(OS), data de realização da OS, código de identificação e especialidade do profissional que solicitou o exame, código de identificação do indivíduo, sexo, data de nascimento e dosagem de creatinina sérica.

Os critérios de inclusão utilizados foram: idade acima de 18 anos, com registros adequados de identificação, sexo, data de nascimento, data da realização do exame e dosagem de creatinina sérica.

A creatinina sérica no referido laboratório é determinada utilizando-se o método cinético-colorimétrico, no aparelho BECKMAN CX4. A faixa de normalidade varia de 0,4 a $1,3 \mathrm{mg} / \mathrm{dl}$.

Iniciamos procedendo ao cálculo da filtração glomerular dos indivíduos submetidos à dosagem de creatinina sérica no período de janeiro de 2004 a dezembro de 2005. A FG foi estimada pela equação do estudo MDRD (Modification of diet in renal disease) na sua versão simplificada, que leva em consideração as variáveis creatinina sérica (SCR), idade (em anos) e sexo:

$\mathrm{FG}\left(\mathrm{ml} / \mathrm{min} / 1,73 \mathrm{~m}^{2}\right)=186 \times(\mathrm{scr})^{-1,154} \times(\text { idade })^{-0,203} \times 0,742(\mathrm{se}$ mulher) $x \mathrm{I}, 2 \mathrm{I}$ (negro americano)

Esta equação utiliza um fator de correção para negros americanos, que não será utilizada neste estudo devido ao inexpressivo número de indivíduos com esta característica racial entre a população brasileira. A fórmula escolhida tem como vantagem fornecer um ajuste para variações substanciais de fatores que interferem na produção de creatinina como idade, sexo e superfície corporal|'
ADRC foi reconhecida e classificada nos estágios 3,4 ou 5 , segundo as diretrizes propostas pelo $\mathrm{K} / \mathrm{DOQI}$ (2002), ou seja, indivíduos que apresentaram $\mathrm{FG}<60 \mathrm{ml} / \mathrm{min} / \mathrm{l}, 73 \mathrm{~m}^{2}$ em dois ou mais registros, em intervalo igual ou superior a três meses foram considerados portadores da DRC e estagiados por meio da FG mais baixa.

Aqueles que apresentaram apenas uma $\mathrm{FG}<60 \mathrm{ml} / \mathrm{min} / \mathrm{l}, 73 \mathrm{~m}^{2}$ nos anos de 2004 e 2005 foram pesquisados nos registros de 1995 a 2003 sobre alterações anteriores. Os que se mantiveram com apenas uma FG $<60$ ou com intervalo inferior a três meses foram considerados como possíveis portadores de uma alteração renal transitória por ocasião do exame. Aqueles com FG> $60 \mathrm{ml} / \mathrm{min} / \mathrm{l}, 73 \mathrm{~m}^{2}$ foram considerados sem evidência de DRC.

O estudo foi aprovado pelo Comitê de Ética em Pesquisa Médica da Universidade Federal de Juiz de Fora.

A análise estatística foi realizada utilizando-se técnicas descritivas e exploratórias. As variáveis quantitativas foram representadas pela média e desvio padrão; mediana, mínima e máxima. As variáveis qualitativas por meio de percentagem. A correlação entre variáveis foi realizada por meio da medida de razão de chance $(\mathrm{RC})$ e risco relativo (RR). Os intervalos de confiança destas medidas foram calculados para um nível de significância de 95\%. Foram utilizados os programas SPSS versão I4.0 e EPI INFO 2002.

\section{Resultados}

O laboratório realizou e disponibilizou 214.576 dosagens de creatinina sérica, no período de janeiro de 1995 a dezembro de 2005, para que os objetivos deste estudo fossem alcançados. Para nossa primeira análise, selecionamos as dosagens de creatinina realizadas em 2004 e 2005, num total de 49.916 registros. Destes, 39.795 contemplaram os critérios de inclusão, correspondendo a 24.248 indivíduos.

O banco de dados para elaboração do presente estudo foi composto por registros de 24.248 indivíduos sendo I4.456 (59,6\%) do sexo feminino e 9.792 (40,4\%) do sexo masculino. A média de exames realizados por indivíduos foi de I,64, não havendo diferença significativa entre os sexos. A idade média dos participantes foi de 48,74 anos $( \pm 14,99)$ com uma amplitude de variação de 18 a 91 anos. No sexo feminino, a idade média foi de 48,99 anos $( \pm$ |4,86) e 48,37 ( \pm |4,85) no sexo masculino.

\section{Avaliação das dosagens de creatinina sérica realizadas em 2004 e 2005}

O valor médio da creatinina sérica foi de $1,0 \mathrm{mg} / \mathrm{dl}( \pm 0,4)$, sendo $0,9( \pm 0,4)$ para o sexo feminino e $1,1( \pm 0,4)$ para o sexo masculino e a amplitude de variação foi de 0,4 a $12,5 \mathrm{mg} / \mathrm{dl}$. Foram encontrados 760 indivíduos $(3,1 \%)$ acima do ponto de corte para normalidade referenciado pelo laboratório, ou seja, acima de I,3 mg/d, sendo 246 $(1,7 \%)$ do sexo feminino e 514 (5,2\%) do sexo masculino, $462(6,9 \%)$ acima de 60 anos, $298(1,7 \%)$ abaixo de 60 anos, 139 (3,4\%) em mulheres acima de 60 anos, 107 (1\%) em mulheres abaixo de 60 anos, 323 (I2,4\%) homens acima de 60 anos e 19| (2,7\%) homens abaixo de 60 anos. Foram encontradas diferenças significativas na análise comparativa entre sexos e faixas etárias, com uma chance 3,3 vezes maior de hipercreatinemia entre os indivíduos acima de 60 anos e 
BASTOS RMR ET AL.

\begin{tabular}{|c|c|c|c|c|c|}
\hline \multicolumn{6}{|c|}{ Tabela I - Média de creatinina e razão de chance (RC) para associaçōes entre variáveis selecionadas e hipercreatinemia } \\
\hline & & Creatinina (mg/dl) & & reatine & \\
\hline Variáveis & $\mathrm{n}$ & Médiae desvio padrão & $n(\%)$ & RC & IC95\% \\
\hline$\overline{G o b d}$ & 24.248 & $1,0 \pm 0,4$ & $760(3,1 \%)$ & & \\
\hline $\begin{array}{l}\text { Sexo } \\
\text { Feminino } \\
\text { Masculino }\end{array}$ & $\begin{array}{l}14.456 \\
9.792\end{array}$ & $\begin{array}{l}0,9 \pm 0,3 \\
1,1 \pm 0,4\end{array}$ & $\begin{array}{l}246(1,7 \%) \\
514(5,2 \%)\end{array}$ & 3,2 & 2,$7 ; 3,7$ \\
\hline $\begin{array}{l}\text { Faixa etária } \\
<60 \text { anos } \\
\geq 60 \text { anos }\end{array}$ & $\begin{array}{c}17.589 \\
6.659\end{array}$ & $\begin{array}{l}1,0 \pm 0,5 \\
1,1 \pm 0,5\end{array}$ & $\begin{array}{l}289(1,7 \%) \\
462(6,9 \%)\end{array}$ & 4,3 & 3,$7 ; 5,0$ \\
\hline $\begin{array}{l}\text { Faixa etária por sex } \\
\geq 60 \text { anos Feminino } \\
\geq 60 \text { anos Masculino } \\
<60 \text { anos Feminino } \\
<60 \text { anos Masculino }\end{array}$ & $\begin{array}{l}4.060 \\
2.599 \\
10.396 \\
7.193\end{array}$ & $\begin{array}{l}1,0 \pm 0,3 \\
1,2 \pm 0,6 \\
0,9 \pm 0,3 \\
1,0 \pm 0,3\end{array}$ & $\begin{array}{c}139(3,4) \\
323(12,4 \%) \\
107(1 \%) \\
191(2,7)\end{array}$ & $\begin{array}{l}4,0 \\
2,6\end{array}$ & $\begin{array}{l}3,3 ; 4,9 \\
2,0 ; 3,2\end{array}$ \\
\hline
\end{tabular}

destaque para os homens cuja chance de apresentarem hipercreatinemia foi quatro vezes a apresentada por mulheres nesta faixa etária (Tabela I).

\section{Avaliação da filtração glomerular em 2004 e 2005}

O valor médio da FG foi de $78 \mathrm{~m} / / \mathrm{min} / \mathrm{l}, 73 \mathrm{~m}^{2}$, sendo $75 \mathrm{ml} / \mathrm{min} /$ I,73m² no sexo feminino e $83 \mathrm{ml} / \mathrm{min} / \mathrm{l}, 73 \mathrm{~m}^{2}$ no sexo masculino. $\mathrm{Na}$ Tabela 2 apresentamos distribuição dos indivíduos em intervalos da Filtração Glomerular sem considerar a cronicidade da alteração.

\section{Condições de morbidade, de acordo com a filtração glomerular}

Aqueles que apresentaram apenas uma $\mathrm{FG}<60 \mathrm{ml} / \mathrm{min} / 1,73 \mathrm{~m}^{2}$ (974 indivíduos) ou duas $F G<60 \mathrm{ml} / \mathrm{min} / 1,73 \mathrm{~m}^{2}$ em intervalo inferior a três meses (49 indivíduos), no período de janeiro de 2004 a dezembro de 2005, foram pesquisados em registros de anos anteriores (1995 a 2003) para a confirmação de cronicidade da disfunção, o que foi obtido em 346 indivíduos. As condições de morbidade foram assim definidas:

Alteração renal transitória: encontramos 683 indivíduos $(2,8 \%)$ que apresentaram apenas um registro de $F G<60 \mathrm{ml} / \mathrm{min} / 1,73 \mathrm{~m}^{2}$ ou cujos registros das $F G<60 \mathrm{ml} / \mathrm{min} / 1,73 \mathrm{~m}^{2}$ ocorreram em intervalo inferior a três meses.

Doentes renais crônicos: aqueles que apresentaram FG $<60 \mathrm{ml} /$ $\mathrm{min} / 1,73 \mathrm{~m}^{2}$ em pelo menos dois registros, em intervalo igual ou superior a três meses, totalizando 2333 (9,6\%), distribuídos em 2.183 (9,0\%), 90 (0,4\%) e 60 (0,2\%), nos estágios 3, 4 e 5, respectivamente.

Sem evidências de DRC ou normais: aqueles comFG $>60 \mathrm{~m} / \mathrm{min} /$ I,73m² num total de 21.232 indivíduos (87,6\%).

\section{Avaliação dos indivíduos com doença renal crônica e fatores associados \\ Foram identificados 2333 indivíduos com DRC, configurando uma prevalência global de 9,6\%. No estágio 3, foram encontrados 2.183 (93,6\%); no estágio 4, identificamos 90 (3,9\%) indivíduos e 60 (2,6\%) no estágio 5.}

\begin{tabular}{lccc}
\hline \multicolumn{4}{c}{ Tabela2- Distribuiçãoporintervalos dafiltraçãoglomerular } \\
\hline $\begin{array}{l}\text { Intervalosda } \\
\text { filtraçãoglomerular }\end{array}$ & $\mathbf{N}$ & Frequência & $\begin{array}{l}\text { Frequência } \\
\text { acumulada }\end{array}$ \\
\hline$\left(\mathrm{m} / / \mathrm{min} / /, 73 \mathrm{~m}^{2}\right)$ & & & \\
$\geq 90$ & 5.579 & 23,0 & 23,0 \\
$60-89$ & 15.653 & 64,6 & 87,6 \\
$30-59$ & 2.822 & 11,6 & 99,2 \\
$\mid 5-29$ & 117 & 0,5 & 99,7 \\
$<15$ & 77 & 0,3 & 100,0 \\
Total & 24.248 & 100 & 100,0 \\
\hline
\end{tabular}

Tabela 3 - Razãode chance ( $R C)$ para associações entre variáveis selecionadase prevalência da doença renal crônica

\begin{tabular}{|c|c|c|c|c|}
\hline Variáveis & $n$ & DRC(\%) & RC & IC95\% \\
\hline $\begin{array}{l}\text { Sexo } \\
\text { Feminino } \\
\text { Masculino }\end{array}$ & $\begin{array}{l}24.248 \\
14.456 \\
9.792\end{array}$ & $\begin{array}{c}1.768(12,2 \%) \\
565(5,8 \%)\end{array}$ & 2,3 & 2,$1 ; 2,5$ \\
\hline $\begin{array}{l}\text { Idade } \\
\geq 60 \text { anos } \\
<60 \text { anos }\end{array}$ & $\begin{array}{l}24.248 \\
6,659 \\
17.589\end{array}$ & $\begin{array}{c}1.676(25,2 \%) \\
657(3,7 \%)\end{array}$ & 8,7 & 7,$9 ; 9,5$ \\
\hline $\begin{array}{l}\text { Faixa etária por sexo } \\
\geq 60 \text { anos, Feminino } \\
\geq 60 \text { anos, Masculino } \\
<60 \text { anos, Feminino } \\
<60 \text { anos, Masculino }\end{array}$ & $\begin{array}{l}24.248 \\
4.060 \\
2.599 \\
10.396 \\
7.193\end{array}$ & $\begin{array}{c}1.241(30,6 \%) \\
435(16,7 \%) \\
527(5,1 \%) \\
130(1,8 \%)\end{array}$ & $\begin{array}{l}2,2 \\
2,9\end{array}$ & $\begin{array}{l}1,9 ; 2,5 \\
2,4 ; 3,5\end{array}$ \\
\hline $\begin{array}{l}\text { Creatinina } \\
\text { Elevada } \\
\text { Normal }\end{array}$ & $\begin{array}{l}24.248 \\
760 \\
23.488\end{array}$ & $\begin{array}{l}587(77,2 \%) \\
1.746(7,4 \%)\end{array}$ & 42,2 & 35,$4 ; 50,4$ \\
\hline $\begin{array}{l}\text { Creatinina normal } \\
\text { Feminino } \\
\text { Masculino } \\
\geq 60 \\
<60\end{array}$ & $\begin{array}{l}14.210 \\
9.278 \\
6197 \\
17.291\end{array}$ & $\begin{array}{l}1.57 \mid(\mid 1,1 \%) \\
175(1,9 \%) \\
1304(21 \%) \\
442(2,6 \%)\end{array}$ & 6,47 & $\begin{array}{l}5,5 ; 7,6 \\
9, \mid \text { a | | ,4 }\end{array}$ \\
\hline
\end{tabular}


Nos estágios I e 2, avaliados pela persistência da proteinúria, foram identificados 18 indivíduos, um no estágio I (FG acima de $90 \mathrm{ml} / \mathrm{min}$ / $1,73 \mathrm{~m}^{2}$ ) e 17 no estágio 2 (FG acima de 60 e abaixo de $90 \mathrm{ml} / \mathrm{min} /$ $1,73 \mathrm{~m}^{2}$ ). Tais indivíduos não foram incluídos neste estudo.

$\mathrm{Na}$ Tabela 3, apresentamos as razões de chances entre a DRC e variáveis selecionadas. Observamos diferenças significativas na análise comparativa entre as prevalências da DRC por sexo, faixa etária e na avaliação quanto à ausência ou presença de hiperuricemia. A chance de $D R C$ apresenta-se significativamente maior $(R C=2,3)$ no sexo feminino. Esta diferença se mantém nas mulheres acima de 60 anos $(\mathrm{RC}=2,2)$. Já em indivíduos com idade inferior a 60 anos, a chance de maior prevalência da DRC encontra-se entre os homens $(R C=2,9)$.

\section{Discussão}

O interesse pelo presente estudo partiu da possibilidade de demonstrar uma metodologia de baixo custo para o rastreamento de indivíduos portadores da DRC com a relevância de caracterizar a cronicidade da doença, negligenciada em vários estudos a despeito das diretrizes atuais para diagnóstico da DRC. A prevalência de 9,6\% da DRC em nossos resultados, considerando a persistência da alteração da FG por pelo menos três meses, foi 2,8\% menor do que as alterações da FG evidenciadas neste estudo, se observadas em uma única medida (I2,4\%).

A população estudada foi constituída pelos indivíduos adultos submetidos a exames em um laboratório privado e não representa, portanto, a população do município. Embora o banco de dados disponibilizado pelo laboratório tenha se mostrado consistente e completo este estudo traz as limitações inerentes à utilização de dados secundários. Por exemplo, destacamos a dificuldade de trabalhar com a variável indicação clínica para a realização dos exames, que permitiria identificar o perfil de morbidade da população estudada e ainda a ausência de exames de proteinúria concomitante às dosagens de creatinina, no mesmo indivíduo, impossibilitando a determinação da prevalência da DRC nos seus estágios iniciais.

Nossos resultados mostram-se concordantes com estudos transversais de base populacional com a mesma faixa etária (acima de 18 anos), a exemplo do estudo realizado por Chadban et al. (2003) que estimou uma prevalência de $10,9 \%, 0,3 \%$ e 0, $1 \%$ da DRC nos estágios 3,4 e 5 , respectivamente, semelhante aos $9,0 \%, 0,4 \%$ e $0,2 \%$ observados neste estudo ${ }^{17}$.

A prevalência expressiva da DRC em indivíduos com idade acima de 60 anos corrobora diversos estudos que evidenciam a idade avançada como fator de risco já bem estabelecido para a doença. Os 25,2\% de prevalência da DRC acima de 60 anos, evidenciados neste estudo, aproximam-se dos 28,9\% obtidos por Swedko e Clark (2003) em indivíduos com idade acima de 65 anos ${ }^{18}$.

A diferença observada entre os sexos feminino (12,2\%) e masculino $(5,8 \%)$ concorda com Viktorsdottir et al. (2005) que, nos estágios 3,4 e 5 , citam prevalências de $11,6 \%$ e 4,7\% nos sexos feminino e masculino, respectivamente ${ }^{19}$.

A prevalência de 3,1\% de hipercreatinemia (I,7\% no sexo feminino e $5,2 \%$ no sexo masculino) em nossos resultados foi a mesma encontrada por Lessa (2004) em Salvador (I,6\% no sexo feminino e 5,2\% no sexo masculino $)^{5}$. A predominância da hipercreatinemia nas idades mais avançadas e no sexo masculino concorda com os resultados de Passos et al. (2003) e confirma dados da literatura ${ }^{14,20}$.

Verifica-se que, se por um lado a prevalência de hipercreatinemia foi maior no sexo masculino, a alteração da filtração glomerular, indicativa da DRC, foi maior no sexo feminino. Tal fato reforça a importância de utilizar a FG e não a dosagem de creatinina isoladamente na avaliação da função renal. Alem disso, a presença da doença renal crônica em 7,4\% dos indivíduos com creatinina normal (1 I, I\% entre as mulheres e 1,9\% entre os homens) confirma a inadequabilidade da hipercreatinemia como critério isolado para o rastreamento da disfunção renal, principalmente no sexo feminino, conforme demonstrado por Pinto et al., 200421.

\section{Conclusão}

Conhecer a prevalência da endemia da DRC é uma questão desafiadora e vem sendo desenvolvida por meio de estimativas que respaldam a elaboração de estratégias para o rastreamento da doença. Os resultados do presente estudo evidenciam a prevalência de 9,6\% da DRC não apenas como um indicador epidemiológico, mas demonstram um aspecto operacional alternativo para otimizar a capacidade de detecção dos casos e permitem sugerir a inclusão do cálculo da filtração glomerular, como um dado complementar aos resultados das dosagens de creatinina sérica fornecidos pelos laboratórios. Tal medida permitiria a visualização imediata da disfunção renal estimulando uma conduta adequada para o diagnóstico e tratamento precoce.

Este trabalho contribui para fundamentar novas propostas à rotina de rastreamento da DRC, para fornecer subsídios para estudos dos fatores relacionados à doença renal e para a redução da lacuna hoje existente na literatura sobre a doença renal crônica, no Brasil.

\section{Agradecimentos}

Agradecemos aos diretores do laboratório pela disponibilização do banco de dados e ao Sr. Flávio Leite Januário responsável técnico pelos registros utilizados para a realização do presentetrabalho.

\section{Conflito de interesse: não há}

\section{SUMMARY}

\section{Prevalence of Chronic kidney disease, stages 3, 4 and 5 in} ADULTS

INTRODUCTION. Strategies thatoptimize early diagnosis of chronickidney disease (CKD) are paramount to decrease progression of the disease and the burden of patients needing renal replacement therapy.

OBIECTIVE. The aim ofthisstudy wasto determine the prevalence of CKD stage 3,4 and 5 in people submitted to dosage of serum creatinine due to different causes, employing a dataset from a private laboratory of the city of Juiz de Fora comprising the years 2004 and 2005.

METHODS. Diagnosis and staging of CKD were based upon glomerular filtration rate (GFR) estimated from serum creatinine as recommended by the KDOQI of the National Kidney Foundation and the Brazilian Society of Nephrology. REsuLTS: Prevalence of CKD stage 3, 4and 5 was of $9.6 \%$, with $12.2 \%, 5.8 \%, 25.2 \%$ and $3.7 \%$ among women, men, people $>60$ and $\leq 60$ years of age, respectively. 
CONCLUSION. Prevalence of CKD found in our study may not only be interpreted as an epidemiologic indicator, but also discloses an alternative operational strategy to identify the disease. Furthermore it supports a proposal to include the estimation of GFR from serum creatinine in the laboratory report as an important and simple tool for early diagnosis of CKD. [Rev Assoc Med Bras 2009; 55(I): 40-4]

KEY WORDS: Chronic kidney disease. Glomerular filtration. Prevalence. Early diagnosis.

\section{REFERÊNCIAS}

I. Atkins RC. The epidemiology of chronic kidney disease. Kidney International 2005; 67 (94), 14-18.

2. Beagleholer R, Yach D. Globalisation and the prevention and control of non-comunicable disease: the neglected chronic diseases of adult. Lancet 2003; 22: 1763-1764.

3. Armstrong T, Bonita R. Capacity building for an integrated noncommunicable disease risk factor surveillance system in developing countries. Ethnicity and Disease 2003; 13(2): 13-18.

4. Yach D, Hawkes C, Gould CL, Hofman KJ. The global burden of chronic diseases: Overcoming impediments to prevention and control. JAMA 2004: 291: 2616-2622.

5. Lessa I. Doenças Crônicas não Transmissíveis no Brasil: um desafio para a complexa tarefa da vigilância. Ciência e Saúde Coletiva 2004; 9(4): $931-943$

6. Kiberd BA, Clase CM. Cumulative risk for developing end-stage renal disease in the US population. J. Am. Soc. Nephrol 2002; 13:1635- 1644.

7. Berthoux F, Jones E, Geller R, Mendel S, Saker L, Briggs D, Epidemiological data of treated end-stage renal failure in the European Union (EU) during the year 1995: report of the European Renal Association Registry and the National Registries. Nephrol Dial Transplan 1999; 14: 2332-42.

8. Locatelli F, Pozzoni P, Tentori F, Del Vecchio L, Epidemiology of cardiovascular risk in patients with chronic kidney disease. Nephrol Dial Transplant 2003; 7: 2-9.

9. Coresh J, Wei GL, McQuillan G, Brancati F, Levey AS, Jones C, et al., Prevalence of high blood pressure and elevated serum creatinine level in the United States: findings from the tirad National Health and Nutrion Examination Survey (1988-1994). Archives Intern Med 200/; 161: 1207- 16

10. Levin A, Identification of patients and risk factors in chronic kidney disease- Evaluatting risk factors and therapeutic strategies. Nephrol Dial Transplant $200 /$; 16(7): 57-60
I I. Sarnak MJ, Levey AS, Schoolwerth AC, Coresh J, Culleton B, et al.: Kidney Disease as risk factor for development of cardiovascular disease. A Statement from the American Heart Association Councils on Kidney in Cardiovascular Disease, High Blood Pressure Research, Clinical, Cardiology, Epidemiology and Prevention. Circulation 2003;108: $2154-74$.

12. Abreu PF, Ramos LR, Sesse R. Abnormalities of renal function in the elderly. Geriatr Nephrol Urol 1999; 9(3): I5।-145.

13. Lopes LMV, Detecção de Doenças Renais: estudo populacional em um bairro da cidade de Recife-PE, Tese apresentada à Universidade Federal de São Paulo- Escola Paulista de Medicina, para obtenção do Título de Doutor em Medicina 2001;1-96.

I 4. Passos VM, Barreto SM, Lima-Costa MF, Detection of renal dysfunction based on serum creatinine levels in a Brazilian community: Bambuí Healthand Agein Study. Braz J Med Biol Res 2003; 36: 393-40I.

|5. K/DOQI clinical practice guidelines for Chronic kidney disease: evaluation, classification and stratification. American Journal Kidney Disease 2002; 39 ( I): I-266.

16. Romão Jr JE: Diretrizes Brasileiras da Doença Renal Crônica. Doença Renal Crônica: definição, epidemiologia e classificação. Jornal Brasileiro de Nefrologia 2004; 26 (3): 4 - 5.

17. Chadban S, Briganti E, Kerr P, et al., Prevalence of kidney damage in Australian adults: The AusDiab Kidney Study. J. Am. Soc. Nephrol 2003, $14: 131-138$

18. Swedko PJ, Clark HD, Paramsothy K, Akbari A. Serum creatinine is an inadequate screening test for renalfailure in elderly patients. Arch Inter Med 2003; 163: 356-360

19. Viktorsdottir O, Palsson R, Adresdottir MB, Asperlund T, Gudnason $\mathrm{V}$, Indridason OS. Prevalence of chronic kidney disease based on estimated glomerular filtration rate and proteinuria in Iceland adults. Nephrol Dial Transplant 2005; 20: 1799-1807.

20. Culleton BF, Larson MG, Evans JC, Wilson PW, Barret BG, et al., Prevalence and correlates of elevated serum creatinine levels: the Frangmingham Heart Study. Archives Intern Méd 1999;159: 1785-90.

21. Pinto PS, Silva FJ, Munch ECSM, Chaoubah A, Bastos RV, Andrade LCF, Bastos MG. Inadequabilidade da Creatinina Sérica na Identificação Precoce da Disfunção Glomerular. Jornal Brasileiro de Nefrologia 2004,26 (4):196-20I.

Artigo recebido: 20/08/07

Aceito para publicação: 09/06/08 